\title{
ADJUNCTION INEQUALITY FOR REAL ALGEBRAIC CURVES
}

\section{G. MiKhalkin}

The zero set of a real polynomial in two variable is a curve in $\mathbb{R}^{2}$. For a generic choice of its coefficients this is a non-singular curve, a collection of circles and lines properly embedded in $\mathbb{R}^{2}$. What topological arrangements of these circles and lines appear for the polynomials of a given degree? This question arose in the 19th century in the works of Harnack and Hilbert and was included by Hilbert into his 16th problem. Several partial results were obtained since then (see [5], [1], [7], [8]). However the complete answer is known only for polynomials of degree 5 (see [6]) or less. The paper presents a new partial result toward the solution of the 16th Hilbert problem.

A consequence of the main theorem of the paper implies that the zero set of a polynomial of degree 7 can not be isotopic to a curve pictured on Fig. 1 (contrary to the curve pictured on Fig. 2). The realizability of Fig. 1 does not contradict to the previously known restrictions. The proof of the main theorem makes use of the proof by Kronheimer and Mrowka [3] of the Thom conjecture in $\mathbb{C} P^{2}$.

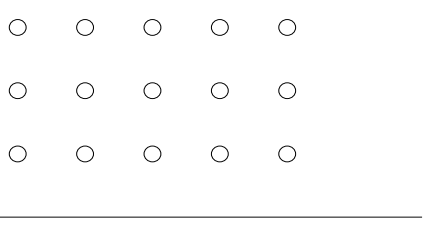

Figure 1. This picture is not isotopic to a curve of degree 7.

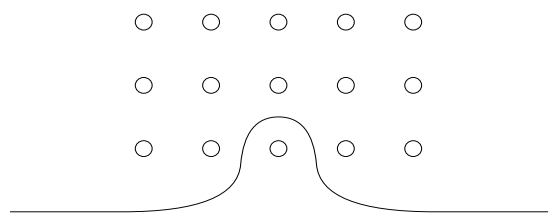

Figure 2. This picture is isotopic to a curve of degree 7.

Received September 9, 1996.

Research at MSRI supported in part by NSF grant DMS-9022140. 


\section{Statement of the theorem}

Let $f$ and $g$ be two real homogeneous polynomials in two variables of degrees $m$ and $n, m \geq n, m \equiv n(\bmod 2)$. Let

$$
\begin{aligned}
& \mathbb{R} A=\left\{\left(x_{0}: x_{1}: x_{2}\right) \in \mathbb{R} P^{2} \mid f\left(x_{0}, x_{1}, x_{2}\right)=0\right\}, \\
& \mathbb{R} B=\left\{\left(x_{0}: x_{1}: x_{2}\right) \in \mathbb{R} P^{2} \mid g\left(x_{0}, x_{1}, x_{2}\right)=0\right\},
\end{aligned}
$$

be the corresponding curves in $\mathbb{R} P^{2}$ and let $\mathbb{C} A$ and $\mathbb{C} B$ be the corresponding curves in $\mathbb{C} P^{2}$. If the coefficients of $f$ and $g$ are generic then $\mathbb{C} A$ and $\mathbb{C} B$ are transverse smooth submanifolds of $\mathbb{C} P^{2}$. We call such curves non-singular. The real parts $\mathbb{R} A$ and $\mathbb{R} B$ of non-singular curves are transverse smooth submanifolds of $\mathbb{R} P^{2}$.

The complex conjugation involution conj : $\left(x_{0}: x_{1}: x_{2}\right) \rightarrow\left(\bar{x}_{0}: \bar{x}_{1}: \bar{x}_{2}\right)$ acts on $\mathbb{C} P^{2}, \mathbb{C} A$ and $\mathbb{C} B$ fixing $\mathbb{R} P^{2}, \mathbb{R} A$ and $\mathbb{R} B$. Note that $\mathbb{C} A-\mathbb{R} A$ is either connected (then $\mathbb{R} A$ is called of type $I I$ ) or consists of two components interchanged by conj (then $\mathbb{R} A$ is called of type $I$ ) since $\mathbb{C} A /$ conj is connected and $\partial \mathbb{C} A /$ conj $=\mathbb{R} A$. If $\mathbb{R} A$ is of type I then following [7] we equip $\mathbb{R} A$ with complex orientation, i.e. with the boundary orientation induced from one of the components of $\mathbb{C} A-\mathbb{R} A$. Because of ambiguity in the choice of the component of $\mathbb{C} A-\mathbb{R} A$ this orientation is defined up to simultaneous reversion of the orientations of all the components of $\mathbb{R} A$.

Let $C \subset \mathbb{R} P^{2}$ be an oriented null-homologous topologically embedded (multicomponent) curve. Following [8] we define a locally constant function called the index

$$
\mid \text { ind } \mid: \mathbb{R} P^{2}-C \rightarrow \mathbb{Z}
$$

in the following way. The index of the non-orientable component of $\mathbb{R} P^{2}-C$ is zero. The complement of the non-orientable component is a disjoint union of disks. A regular neighborhood $N$ of each of these disks is $\mathbb{R}^{2}$ and in $N$ there is a well-defined index of a point with respect to $C \cap N$ once we pick an orientation of $N$. We define ind $\left.\right|_{N-C}$ as the absolute value of the index in $N$.

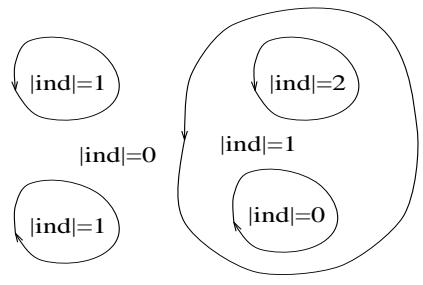

Figure 3. Function $\mid$ ind $\mid$.

For each locally constant function $F: \mathbb{R} P^{2}-C \rightarrow \mathbb{R}$ we define

$$
\int_{\mathbb{R} P^{2}} F d \chi=\sum_{G} F(G) \chi(G),
$$


over all components $G$ of $\mathbb{R} P^{2}-C$ ( $\chi$ denotes the Euler characteristic).

Let $\mathbb{R} A$ and $\mathbb{R} B$ be the curves of type I equipped with the complex orientations. We associate the following curve $C$ to $\mathbb{R} A$ and $\mathbb{R} B$. Take $\mathbb{R} A \cup \mathbb{R} B$ and smooth all the points of intersection according to their orientations choosing any pattern of Fig. 4 (cf. smoothing in [4]). (Recall that $\mathbb{R} A$ and $\mathbb{R} B$ are transverse since we assume that coefficients of $f$ and $g$ are generic and $C$ is homologous to zero since $m \equiv n(\bmod 2))$.
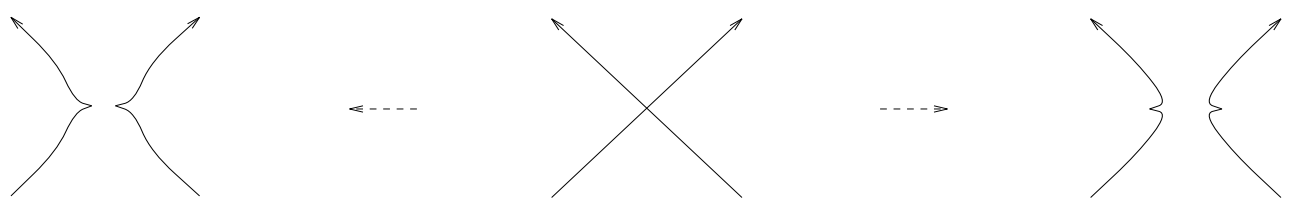

Figure 4. Smoothings of a double point.

Let $G$ be a domain in $\mathbb{R} P^{2}$ such that $\partial G$ is a component of $C$. Let $c_{\text {out }}\left(c_{\text {in }}\right)$ be the number of the outward (inward) with respect to $G$ cusps on the boundary of $G$. Let $\chi^{\prime}(G)=\chi(G)-\frac{1}{2}\left(c_{\text {out }}-c_{\text {in }}\right)$ (cf. Remark 1.3 of [4]). Define "the negative variation" of $\chi^{\prime}$ by the following rules. For a connected $G$ let $\chi_{-}^{\prime}(G)=-\chi^{\prime}(G)$ if $\chi^{\prime}(G)<0$ and $\chi_{-}^{\prime}(G)=0$ if $\chi^{\prime}(G) \geq 0$. For a disconnected $G$ define $\chi_{-}^{\prime}(G)$ as the sum of $\chi^{\prime}$ over all components of $G$. Define

$$
\Xi=\sum_{s=1}^{\infty} s \chi_{-}^{\prime}\{|\operatorname{ind}(x)|>s\} .
$$

Note that $\Xi=0$ if $\mid$ ind $\mid<2$ on all components of $\mathbb{R} P^{2}-C$ of negative Euler characteristic. This will be the case for our applications. Let $d$ be the number of the intersection points of $\mathbb{R} A$ and $\mathbb{R} B$.

\section{Theorem 1.}

$$
\int_{\mathbb{R} P^{2}} \mid \text { ind } \mid d \chi \leq \frac{(m+n)^{2}}{4}-3 n+d+2 \Xi .
$$

Note that there is ambiguity in choosing the complex orientations of $\mathbb{R} A$ and $\mathbb{R} B$ and in smoothing of double points of $\mathbb{R} A \cup \mathbb{R} B$. The inequality of the theorem holds for any choice of the complex orientation and smoothings.

Remark. If $\mathbb{R} A$ is a non-singular curve of type I of degree $m$ then the curve obtained by taking $s$ parallel copies of each component of $\mathbb{R} A$ is isotopic to a non-singular curve of type I of degree $s m$. Indeed, if $f$ defines $\mathbb{R} A$ then

$$
f(f-\epsilon h) \ldots(f-(s-1) \epsilon h)=\delta p,
$$

defines the required curve for a choice of real numbers $\epsilon, \delta$ and polynomials $h$ and $p$ of degree $m$ and $s m$. 
This observation combined with the theorem yields additional restrictions on mutual position of two curves of type I. In particular, it allows to use the theorem in the case when the degrees of two curves are of opposite parity.

\section{Proof of Theorem 1}

Consider first the special case when $\mathbb{R} A \cap \mathbb{R} B=\emptyset$. Let $A_{+}$be the component of $\mathbb{C} A-\mathbb{R} A$ which determines the chosen complex orientation of $\mathbb{R} A$ and let $B_{-}$ be the component of $\mathbb{C} B-\mathbb{R} B$ which determines the orientation opposite to the chosen orientation of $\mathbb{R} B$. Let

$$
F^{\prime}=A_{+} \cup B_{-} \cup L,
$$

where $L=\bigcup_{s=1}^{\infty}\{\mid$ ind $\mid \geq s\}$. Note that $F^{\prime}$ makes an integer cycle such that the orientation induced from $F^{\prime}$ on $A_{+}$is holomorphic and the orientation induced from $F^{\prime}$ on $B_{-}$is antiholomorphic.

Let $F$ be the surface smoothly generically immersed into $\mathbb{C} P^{2}$ obtained by smoothing the corners, and then, perturbation of $F^{\prime}$. The double points of $F$ come from two sources, from $A_{+} \cap B_{-}$and from perturbation of $\bigcup_{s=1}^{\infty}\{\mid$ ind $\mid \geq s\}$. Note that the double points coming from $A_{+} \cap B_{-}$are all negative since all points of intersection of $\mathbb{C} A$ and $\mathbb{C} B$ equipped with the holomorphic orientation are positive.

To obtain $F$ out of $F^{\prime}$ we perturb every component $G^{\prime} \subset \mathbb{R} P^{2}$ of $\{\mid$ ind $\mid \geq s\}$, $s>1$, to $G$ along a generic vector field $v$ normal to $G^{\prime}$ but tangent to $\mathbb{C} A \cup \mathbb{C} B$ along $\partial G^{\prime} \subset \mathbb{R} A \cup \mathbb{R} B$ (to keep $F$ a cycle). The field $i v$ is tangent to $G^{\prime}$ and to $\partial G^{\prime}$. The zeroes of $v$ and $i v$ are of opposite index. Therefore (inductively by $s$ ) we can choose the perturbation field $v$ so that $G$ (equipped with the orientation induced from $F$ ) intersects the result of perturbation of $\{\mid$ ind $\mid \geq t\}, t<s$, in $\left|\chi\left(G^{\prime}\right)\right|$ points, which are negative if $\chi\left(G^{\prime}\right)>0$ and positive if $\chi\left(G^{\prime}\right)<0$. Therefore, the total number of positive double points of $F$ is $\Xi$.

Note that

$$
[F]=\frac{m-n}{2}\left[\mathbb{C} P^{1}\right] \in H_{2}\left(\mathbb{C} P^{2}\right),
$$

since $F \cup \operatorname{conj}(F)$ is homologous to $[\mathbb{C} A]-[\mathbb{C} B]=(m-n)\left[\mathbb{C} P^{1}\right]$ and conj acts as $(-1)$ on $H_{2}\left(\mathbb{C} P^{2}\right)$. We need the following strengthening of the Thom conjecture $[3]$

$$
-\chi(F)+2 \Xi \geq \frac{(m-n)^{2}}{4}-3 \frac{m-n}{2} .
$$

This strengthening is implicitly contained in [3]. Indeed, it is proven there that if $H$ is a surface of positive genus smoothly embedded to $\mathbb{C} P^{2} \# \overline{\mathbb{C}} P^{2} \# \ldots \# \overline{\mathbb{C} P} P^{2}$, $H . H \geq 0$ and $H$ is of homology class of an algebraic curve then

$$
-\chi(H) \geq H . H+K . H,
$$



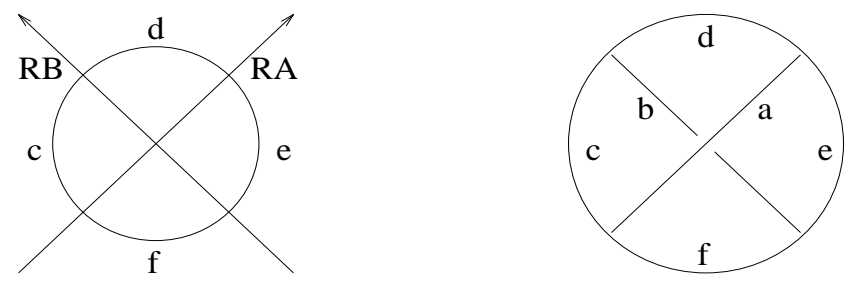

Figure 5. A double point in the real plane and its link in the complexification.

where $K=(-3,1, \ldots, 1) \in H_{2}\left(\mathbb{C} P^{2} \# \overline{\mathbb{C} P} P^{2} \# \ldots \# \overline{\mathbb{C} P} P^{2}\right)$ is the canonical class. The first step to get $H$ out of $F$ is to blow up all the negative double points of $F$. The reason why making a connected sum with $\overline{\mathbb{C}} P^{2}$ allows to get rid of a negative double point is that the intersection of $F$ with a small sphere centered at a double point of $F$ is the Hopf link. If we switch the orientation of one of the component of the negative Hopf link then the result is the positive Hopf link. Note that blowing up the negative points of $F$ does not change $F . F$ and $K . F$, since the resulting surface intersects each exceptional divisor at two points of opposite signs. To get rid of $\Xi$ positive double points of $F$ and obtain $H$ we remove the neighborhood of each positive double point of the surface and glue a handle instead. This increases $-\chi(F)$ by $2 \Xi$. Thus, (2) implies that $-\chi(F)+2 \Xi \geq F . F+K . F=\frac{(m-n)^{2}}{4}-3 \frac{m-n}{2}$. By additivity of $\chi$

$$
\begin{aligned}
& \chi(F)=\chi\left(A_{+}\right)+\chi\left(B_{-}\right)+\chi(L)= \\
& \chi\left(A_{+}\right)+\chi\left(B_{-}\right)+\sum_{s=1}^{\infty} \chi(\mid \text { ind } \mid \geq s)=\chi\left(A_{+}\right)+\chi\left(B_{-}\right)+\int_{\mathbb{R} P^{2}} \mid \text { ind } \mid d \chi .
\end{aligned}
$$

But $\chi\left(A_{+}\right)=\frac{1}{2} \chi(\mathbb{C} A)=-\frac{m^{2}-3 m}{2}$ and $\chi\left(B_{-}\right)=\frac{1}{2} \chi(\mathbb{C} B)=-\frac{n^{2}-3 n}{2}$ by the adjunction formula for the holomorphic curves $\mathbb{C} A$ and $\mathbb{C} B$. Combining this with (1) and (3) we get

$$
\begin{aligned}
\int_{\mathbb{R} P^{2}} \mid \text { ind } \mid d \chi \leq \frac{m^{2}-3 m}{2}+\frac{n^{2}-3 n}{2}-\frac{(m-n)^{2}}{4}+ & 3 \frac{m-n}{2}+2 \Xi \\
& =\frac{(m+n)^{2}}{4}-3 n+2 \Xi .
\end{aligned}
$$

We return to the general case when $\mathbb{R} A$ and $\mathbb{R} B$ intersect in $d$ points. We do the same thing, starting from $F^{\prime}=A_{+} \cup B_{-} \cup \bigcup_{s=1}^{\infty}\{\mid$ ind $\mid \geq s\}$ but we have to pay special attention to smoothing of $F^{\prime}$ near $\mathbb{R} A \cap \mathbb{R} B$. The intersection of $A_{+}$, $B_{-}$and $\mathbb{R} P^{2}$ with a small sphere centered in a point of $\mathbb{R} A \cap \mathbb{R} B$ is pictured on Fig. 5. It consists of 6 arcs, 2 of them, $a$ and $b$ come from the intersection with $A_{+}$and $B_{-}$and 4 of them, $c, d, e, f$, come from the intersection with $\mathbb{R} P^{2}$. For perturbation of $F^{\prime}$ we need to choose such a framing on the circle $a \cup d \cup b \cup f$ that the result of perturbation of $a \cup d \cup b \cup f$ along this framing is not linked 


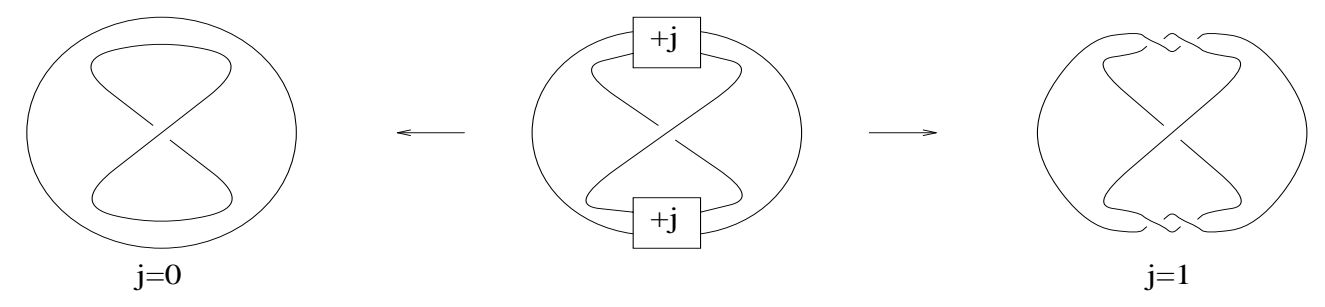

Figure 6. Choosing the framing along $d$ and $f$.

with $c \cup d \cup e \cup f$. The number of (full right) twists $j$ of the framing along $f$ has to be equal to the number of twists along $d$ in order for these circles to be homologically unlinked. These circles are not linked if (and only if) $j=0$ or 1 (see Fig. 6). This corresponds to the framing obtained by multiplication by $i$ of the vector field tangent to the result of smoothing with two inwards or outwards cusps (cf. Fig. 4). Gluing the disk bounded by $a \cup d \cup b \cup f$ along the arcs $f$ and $d$ decreases the Euler characteristic of $F$ by 1 , so the number of the points of $\mathbb{R} A \cap \mathbb{R} B$ appears in the final formula.

\section{Applications}

By the Harnack inequality [2] an affine curve of degree $2 k+1$ has no more then $k(2 k-1)$ ovals.

Proposition 1. If an affine curve of degree $2 k+1$ consists of an arc and $k(2 k-$ 1) ovals with disjoint interiors then each component of the complement of the arc in the affine plane contains at least $\frac{(k-1)(k-2)}{2}$ ovals.

Remark. This proposition is sharp in the following sense. For any $k>0$ there exist affine curves of degree $(2 k+1)$ which consist of $k(2 k-1)$ ovals and an arc where the arc separates $\frac{(k-1)(k-2)}{2}$ ovals from $\frac{(3 k-2)(k+1)}{2}$ ovals. Construction of such curves can be easily extracted from Harnack's construction of curves with $k(2 k-1)$ ovals [2].

Proof. We apply Theorem 1 to the union of the affine curve and the infinite line in $\mathbb{R} P^{2}$. The projectivization of the affine curve is of type I because the curve is extremal for the Harnack inequality (see [7]). Suppose the arc separates $A$ ovals from $k(2 k-1)-A$ ovals. Then for a proper choice of the orientation of the infinite line and the affine curve

$$
\int_{\mathbb{R} P^{2}} \mid \text { ind } \mid d \chi \geq k(2 k-1)-A+1-A,
$$

(the right hand side is the Euler characteristic of the region with $\mid$ ind $\mid=1$, the only possible regions with $\mid$ ind $\mid>1$ are the interiors of some of the $A$ ovals (this depends on their orientation), $d=1$ and $\Xi=0$. Theorem 1 implies now that $1+k(2 k-1)-2 A \leq(k+1)^{2}-3+1$ and, therefore $A \geq \frac{(k-1)(k-2)}{2}$. 
Corollary 1. The curve pictured on Fig. 1 is not isotopic to an affine algebraic curve of degree 7 .

Remark. Degree 7 is the smallest degree when such a restriction appears. For an affine curve of degree 5 which consists of an arc and some ovals there are no constraints on distribution of the ovals between the half-planes (as long as their total number is no more than 6 ).

For even degree $2 k$ Harnack's construction (for the maximal number of ovals) produces a curve $\mathbb{R} P^{2}$ which consists of a non-empty oval, $\frac{k^{2}-3 k+2}{2}$ empty ovals inside the non-empty ovals and $\frac{3 k^{2}-3 k}{2}$ empty ovals outside of the non-empty oval (see Fig. 8 for $k=4$ ). Let $\mathbb{R} A \subset \mathbb{R} P^{2}$ be a curve of degree $2 k$ with such an arrangement of the ovals.

Proposition 2. Not more than $(k-2)$ out of the $\frac{k^{2}-3 k+2}{2}$ interior ovals of $\mathbb{R} A$ may be contained inside an ellipse (a non-singular curve of degree 2 ) in $\mathbb{R} P^{2}$ not intersecting $\mathbb{R} A$.

Remark. This proposition is also sharp. For the Harnack curves of degree $2 k$ we may find ellipses enclosing $(k-2)$ of the interior ovals and not intersecting the curves.

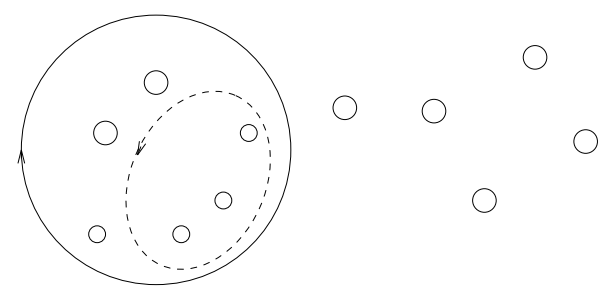

FiguRE 7. Orientations of the ellipse and the non-empty oval of $\mathbb{R} A$.

Proof. Let $L>0$ be the number of the interior ovals inside the ellipse. Apply Theorem 1 to $\mathbb{R} A$ and the ellipse oriented so that the non-empty oval of $\mathbb{R} A$ and the ellipse are oriented in the opposite way (see Fig. 7). We get

$$
\int_{\mathbb{R} P^{2}} \mid \text { ind } \mid d \chi \geq \frac{3 k^{2}-3 k}{2}-\frac{k^{2}-3 k+2}{2}+2 L=k^{2}-1+2 L,
$$

$d=0$ and $\Xi=0$. Therefore, $k^{2}-1+2 L \leq(k+1)^{2}-6$ and $L \leq k-2$.

Corollary 2. It is not possible to separate the 3 interior ovals of a curve of degree 8 with topological arrangement $<18 \sqcup 1<3>>$ from the rest of the ovals by a conic. 
Remark. Theorem 1 generalizes for curves on algebraic surfaces other than $\mathbb{R} P^{2}$. Instead of $m \equiv n(\bmod 2)$ we require that the union of the two curves is nullhomologous in the surface (so that $\mid$ ind $\mid$ is well-defined). The formula then turns to

$$
\int_{\mathbb{R} P^{2}} \mid \text { ind } \mid d \chi \leq \frac{([\mathbb{C} A]+[\mathbb{C} B])^{2}}{4}+K \cdot[\mathbb{C} B]+d+2 \Xi,
$$

where $K$ is the canonical class of the complexification of the surface.

If the geometric genus of the surface is positive then it is not required that the degree of $\mathbb{C} A$ be greater than or equal to the degree of $\mathbb{C} B$.

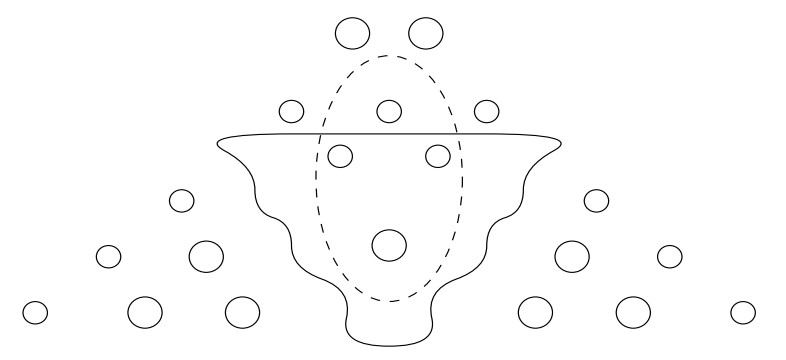

Figure 8. The Harnack curve of degree 8 and a conic.

\section{References}

1. V. I. Arnold, On the distribution of the ovals of real plane curves, involutions of 4dimensional smooth manifolds and the arithmetics of integer-valued quadratic forms, Funktsional. Anal. i Prilozhen. 5 (1971), 1-9.

2. A. Harnack, Über Vieltheiligkeit der ebenen algebraischen Curven, Math. Ann. 10 (1876), 189-199.

3. P. B. Kronheimer and T. S. Mrowka, The genus of embedded surfaces in the projective plane, Math. Res. Lett. 1 (1994), 797-808.

4. G. Mikhalkin and M. Polyak, Whitney formula in higher dimensions, J. Diff. Geom. (to appear).

5. I. G. Petrowsky, On the topology of real plane algebraic curves, Ann. Math. 39 (1938), 187-209.

6. G. M. Polotovskii, Catalog of M-decomposing curves of order 6, Dokl. Akad. Nauk SSSR 236 (1977), 548-551.

7. V. A. Rokhlin, Congruences modulo 16 in Hilbert's sixteenth problem, Funktsional. Anal. i Prilozhen. 6 (1972), 58-64.

8. O. Ya. Viro, The progress in topology of real algebraic varieties over the last six years, Uspekhi Mat. Nauk 41 (1986), 45-67.

Steklov Institute (POMI), Fontanka 27, St.Petersburg, 191011 RUSSiA

Current address: MSRI, 1000 Centennial Dr., Berkeley, CA 94720 USA

E-mail address: mihalkin@pdmi.ras.ru, grisha@msri.org 